\title{
Multidecompositions of the Balanced Complete Bipartite Graph into Paths and Stars
}

\author{
Hung-Chih Lee and Yen-Po Chu \\ Department of Information Technology, Ling Tung University, Taichung 40852, Taiwan \\ Correspondence should be addressed to Hung-Chih Lee; birdy@teamail.ltu.edu.tw
}

Received 30 December 2012; Accepted 30 January 2013

Academic Editors: C. da Fonseca, L. Feng, A. P. Godbole, E. Kiliç, E. Manstavicius, and S. Zhou

Copyright (C) 2013 H.-C. Lee and Y.-P. Chu. This is an open access article distributed under the Creative Commons Attribution License, which permits unrestricted use, distribution, and reproduction in any medium, provided the original work is properly cited.

Let $P_{k}$ and $S_{k}$ denote a path and a star with $k$ edges, respectively. For graphs $F$, $G$, and $H$, a $(G, H)$-multidecomposition of $F$ is a partition of the edge set of $F$ into copies of $G$ and copies of $H$ with at least one copy of $G$ and at least one copy of $H$. In this paper, necessary and sufficient conditions for the existence of the $\left(P_{k}, S_{k}\right)$-multidecomposition of the balanced complete bipartite graph are given.

\section{Introduction}

Let $F, G$, and $H$ be graphs. A $G$-decomposition of $F$ is a partition of the edge set of $F$ into copies of $G$. If $F$ has a $G$ decomposition, we say that $F$ is $G$-decomposable and write $G \mid F$. A $(G, H)$-multidecomposition of $F$ is a partition of the edge set of $F$ into copies of $G$ and copies of $H$ with at least one copy of $G$ and at least one copy of $H$. If $F$ has a $(G, H)$-multidecomposition, we say that $F$ is $(G, H)$ multidecomposable and write $(G, H) \mid F$.

For positive integers $m$ and $n, K_{m, n}$ denotes the complete bipartite graph with parts of sizes $m$ and $n$. A complete bipartite graph is balanced if $m=n$. A $k$-path, denoted by $P_{k}$, is a path with $k$ edges. A $k$-star, denoted by $S_{k}$, is the complete bipartite graph $K_{1, k}$. A $k$-cycle, denoted by $C_{k}$, is a cycle of length $k$.

$P_{k}$-decompositions of graphs have been a popular topic of research in graph theory. Articles of interest include [1-11]. The reader can refer to [12] for an excellent survey of this topic. Decompositions of graphs into $k$ stars have also attracted a fair share of interest. Articles of interest include [13-18]. The study of the $(G, H)$ multidecomposition was introduced by Abueida and Daven in [19]. Abueida and Daven [20] investigated the problem of the $\left(K_{k}, S_{k}\right)$-multidecomposition of the complete graph $K_{n}$. Abueida and Daven [21] investigated the problem of the $\left(C_{4}, E_{2}\right)$-multidecomposition of several graph products where $E_{2}$ denotes two vertex disjoint edges. Abueida and O'Neil [22] settled the existence problem of the $\left(C_{k}, S_{k-1}\right)$ multidecomposition of the complete multigraph $\lambda K_{n}$ for $k=3,4$, and 5. In [23], Priyadharsini and Muthusamy gave necessary and sufficient conditions for the existence of the $\left(G_{n}, H_{n}\right)$-multidecomposition of $\lambda K_{n}$ where $G_{n}, H_{n} \in$ $\left\{C_{n}, P_{n-1}, S_{n-1}\right\}$. Furthermore, Shyu [24] investigated the problem of decomposing $K_{n}$ into $k$-paths and $k$-stars, and gave a necessary and sufficient condition for $k=3$. In [25], Shyu considered the existence of a decomposition of $K_{n}$ into $k$-paths and $k$-cycles and established a necessary and sufficient condition for $k=4$. He also gave criteria for the existence of a decomposition of $K_{n}$ into 3-paths and 3 cycles in [26]. Shyu [27] investigated the problem of decomposing $K_{n}$ into $k$-cycles and $k$-stars and settled the case $k=4$. Recently, Lee [28] established necessary and sufficient conditions for the existence of the $\left(C_{k}, S_{k}\right)$ multidecomposition of a complete bipartite graph.

In this paper, we investigate the problem of the $\left(P_{k}, S_{k}\right)$ multidecomposition of the balanced complete bipartite graph and give necessary and sufficient conditions for such a multidecomposition to exist.

\section{Preliminaries}

For our discussions, some terminologies and notations are needed. Let $G$ be a graph. The degree of a vertex $x$ of $G$, 
denoted by $\operatorname{deg}_{G} x$, is the number of edges incident with $x$. A graph is $r$-regular if each vertex is of degree $r$. The vertex of degree $k$ in $S_{k}$ is called the center of $S_{k}$. Let $V$ and $E$ be subsets of the vertex set and the edge set of $G$, respectively. We use $G[V]$ to denote the subgraph of $G$ induced by $V$ and $G-E$ to denote the subgraph obtained from $G$ by deleting $E$. Suppose that $G_{1}, G_{2}, \ldots, G_{t}$ are edge-disjointgraphs. Then, $G_{1}+G_{2}+\cdots+G_{t}$, or $\sum_{i=1}^{t} G_{i}$, denotes the graph $H$ with vertex set $V(H)=\bigcup_{i=1}^{t} V\left(G_{i}\right)$, and edge set $E(H)=\bigcup_{i=1}^{t} E\left(G_{i}\right)$. Thus, if a graph $H$ can be decomposed into subgraphs $G_{1}, G_{2}, \ldots, G_{t}$, we write $H=G_{1}+G_{2}+$ $\cdots+G_{t}$, or $H=\sum_{i=1}^{t} G_{i}$. Moreover, $\lceil x\rceil$ denotes the smallest integer not less than $x$ and $\lfloor x\rfloor$ denotes the largest integer not greater than $x$. Let $v_{0} v_{1} v_{2}, \ldots, v_{k}$ denote the $k$-path with edges $v_{0} v_{1}, v_{1} v_{2}, \ldots, v_{k-1} v_{k}$, and $\left(v_{1}, v_{2}, \ldots, v_{k}\right)$ denote the $k$ cycle with edges $v_{1} v_{2}, v_{2} v_{3}, \ldots, v_{k-1} v_{k}, v_{k} v_{1}$. Throughout the paper, $(A, B)$ denotes the bipartition of $K_{n, n}$, where $A=$ $\left\{a_{0}, a_{1}, \ldots, a_{n-1}\right\}$ and $B=\left\{b_{0}, b_{1}, \ldots, b_{n-1}\right\}$.

For the edge $a_{i} b_{j}$ in $K_{n, n}$, the label of $a_{i} b_{j}$ is $j-i(\bmod$ $n)$. For example, in $K_{9,9}$ the labels of $a_{3} b_{7}$ and $a_{8} b_{2}$ are 4 and 3 , respectively. Note that each vertex of $K_{n, n}$ is incident with exactly one edge with label $i$ for $i=0,1,2, \ldots, n-1$. Let $G$ be a subgraph of $K_{n, n}$ and $t$ a nonnegative integer. We use $G_{+t}$ to denote the graph with vertex set $\left\{a_{i}: a_{i} \in V(G)\right\} \bigcup\left\{b_{j+t}\right.$ : $\left.b_{j} \in V(G)\right\}$ and edge set $\left\{a_{i} b_{j+t}: a_{i} b_{j} \in E(G)\right\}$, where the subscripts of $b$ are taken modulo $n$. In particular, $G_{+0}=G$.

The following results due to Yamamoto et al. and Parker, respectively, are essential for our discussions.

Proposition 1 (see [18]). Let $m \geq n \geq 1$ be integers. Then, $K_{m, n}$ is $S_{k}$-decomposable if and only if $m \geq k$ and

$$
\begin{array}{cl}
m \equiv 0(\bmod k) & \text { if } n<k, \\
m n \equiv 0(\bmod k) & \text { if } n \geq k .
\end{array}
$$

Proposition 2 (see [7]). There exists a $P_{k}$-decomposition of $K_{m, n}$ if and only if $m n \equiv 0(\bmod k)$, and one of aforementioned (see Table 1) cases occurs.

\section{Main Results}

We first give necessary conditions of the $\left(P_{k}, S_{k}\right)$-multidecomposition of $K_{n, n}$.

Lemma 3. Let $k$ and $n$ be positive integers. If there exists a $\left(P_{k}, S_{k}\right)$-multidecomposition of $K_{n, n}$, then $k \leq n$ and $n^{2} \equiv$ $0(\bmod k)$.

Proof. The result follows from the fact that the maximum size of a star in $K_{n, n}$ is $n$, the size of each member in the multidecomposition is $k$, and $\left|E\left(K_{n, n}\right)\right|=n^{2}$.

We now show that the necessary conditions are also sufficient. Since $P_{k}=S_{k}$ for $k=1,2$, the result holds for $k=1,2$ by Proposition 1 . So it remains to consider the case $k \geq 3$. The proof is divided into cases $n \geq 2 k, n=k$ and $k<n<2 k$, which are treated in Lemmas 4, 5, and 6, respectively.
TABLE 1

\begin{tabular}{lcccc}
\hline Case & $k$ & $m$ & $n$ & Conditions \\
\hline 1 & Even & Even & Even & $k \leq 2 m, k \leq 2 n$, both are not equalities \\
2 & Even & Even & Odd & $k \leq 2 m-2, k \leq 2 n$ \\
3 & Even & Odd & Even & $k \leq 2 m, k \leq 2 n-2$ \\
4 & Odd & Even & Even & $k \leq 2 m-1, k \leq 2 n-1$ \\
5 & Odd & Even & Odd & $k \leq 2 m-1, k \leq n$ \\
6 & Odd & Odd & Even & $k \leq m, k \leq 2 n-1$ \\
7 & Odd & Odd & Odd & $k \leq m, k \leq n$ \\
\hline
\end{tabular}

Lemma 4. Let $k$ and $n$ be positive integers with $n \geq 2 k \geq 6$. If $n^{2} \equiv 0(\bmod k)$, then $K_{n, n}$ is $\left(P_{k}, S_{k}\right)$-multidecomposable.

Proof. Let $n=q k+r$ where $q$ and $r$ are integers with $0 \leq r<$ $k$. Then, $q \geq 2$ from the assumption $n \geq 2 k$. Note that

$$
\begin{aligned}
K_{n, n} & =K_{q k+r, n} \\
& =K_{(q-1) k, n}+K_{k+r, n} .
\end{aligned}
$$

By Proposition 1, $K_{(q-1) k, n}$ is $S_{k}$-decomposable. On the other hand, trivially, $k \leq \min \{k+r, n\}$, and $\left|E\left(K_{k+r, n}\right)\right|=(k+r) n \equiv$ $0(\bmod k)$ from the assumption $n^{2} \equiv 0(\bmod k)$. This implies that $K_{k+r, n}$ is $P_{k}$-decomposable by Proposition 2 . Hence, $K_{n, n}$ is $\left(P_{k}, S_{k}\right)$-multidecomposable.

Lemma 5. Let $k$ be a positive integer with $k \geq 3$. Then, $K_{k, k}$ is $\left(P_{k}, S_{k}\right)$-multidecomposable.

Proof. Note that $K_{k, k}=K_{1, k}+K_{k-1, k}$. Trivially, $K_{1, k}=S_{k}$. On the other hand, $\left|E\left(K_{k-1, k}\right)\right|=(k-1) k \equiv 0(\bmod k)$. Furthermore, $k \leq 2(k-1)-1$ for odd $k$, and $k \leq 2(k-$ $1)=2 k-2$ for even $k$. Hence, $K_{k-1, k}$ is $P_{k}$-decomposable by Proposition 2, and $K_{k, k}$ is $\left(P_{k}, S_{k}\right)$-multidecomposable.

Lemma 6. Let $k$ and $n$ be integers with $3 \leq k<n<2 k$. If $n^{2} \equiv 0(\bmod k)$, then $K_{n, n}$ is $\left(P_{k}, S_{k}\right)$-multidecomposable.

Proof. Suppose that $n=k+r$. Then, $0<r<k$ from the assumption $k<n<2 k$. Let $A_{1}=\left\{a_{0}, a_{1}, \ldots, a_{k-1}\right\}$, $B_{1}=\left\{b_{0}, b_{1}, \ldots, b_{k-1}\right\}, A_{2}=\left\{a_{k}, a_{k+1}, \ldots, a_{k+r-1}\right\}$, and $B_{2}=$ $\left\{b_{k}, b_{k+1}, \ldots, b_{k+r-1}\right\}$. Let $G_{i}=K_{n, n}\left[A_{i} \cup B_{1}\right]$ for $i=1,2$ and $H=K_{n, n}\left[A \cup B_{2}\right]$. Then, $K_{n, n}=G_{1}+G_{2}+H$. Note that $G_{1}$ is isomorphic to $K_{k, k}, H$ is isomorphic to $K_{k+r, r}$, and $G_{2}$ is isomorphic to $K_{r, k}$, which is $S_{k}$-decomposable by Proposition 1. Hence, it is sufficient to show that $G_{1}+H$ is $\left(P_{k}, S_{k}\right)$-multidecomposable.

Let $t=r^{2} / k$. Since $k \mid n^{2}$, we have $k \mid r^{2}$, which implies that $t$ is a positive integer Let $C=$ $\left(b_{0}, a_{0}, b_{1}, a_{1}, \ldots, b_{k-2}, a_{k-2}, b_{k-1}, a_{k-1}\right)$. Then, $C$ is a $2 k$-cycle in $G_{1}$. Let $p=\lfloor t / 2\rfloor$. For odd $t$, define a $k$-path $P$ in $G_{1}$ as follows:

$$
P=\left\{\begin{array}{r}
b_{2 p} a_{0} b_{2 p+1} a_{1} \cdots b_{2 p-1+k / 2} a_{k / 2-1} b_{2 p+k / 2} \\
\text { if } k \text { is even, } \\
b_{2 p} a_{0} b_{2 p+1} a_{1} \cdots b_{2 p+(k-1) / 2} a_{(k-1) / 2} \\
\text { if } k \text { is odd }
\end{array}\right.
$$


where the subscripts of $b$ are taken modulo $k$. Since $t=r^{2} / k$ and $r<k$, we have

$$
t \leq r-1 \leq k-2
$$

Thus, $2 p+1=t \leq k-2$, which implies the labels of the edges in $P$ are $2 p$ and $2 p+1$. Note that for $i=0,1, \ldots, p-1, C_{+2 i}$ is a $2 k$-cycle which consists of all of the edges with labels $2 i$ and $2 i+1$ in $G_{1}$. Thus, $C, C_{+2}, \ldots, C_{+2(p-1)}$ and $P$ are edge-disjoint in $G_{1}$.

Define a subgraph $W$ of $G_{1}$ as follows:

$$
W= \begin{cases}\sum_{i=0}^{p-1} C_{+2 i} & \text { for even } t \\ \sum_{i=0}^{p-1} C_{+2 i}+P & \text { for odd } t \geq 3 \\ P & \text { for } t=1\end{cases}
$$

Since $C_{2 k}$ can be decomposed into 2 copies of $P_{k}$ and $2 p=t$ for even $t$ as well as $2 p+1=t$ for odd $t, W$ can be decomposed into $t$ copies of $P_{k}$. Let $\delta=1$ for even $k$ and $\delta=0$ for odd $k$. Note that for even $t, \operatorname{deg}_{G_{1}-E(W)} a_{i}=k-2 p=k-t$, and for odd $t$,

$$
\begin{aligned}
& \operatorname{deg}_{G_{1}-E(W)} a_{i} \\
& = \begin{cases}k-t-1 & \text { if } i=0,1, \ldots,\left\lceil\frac{k}{2}\right\rceil-2, \\
k-t-\delta & \text { if } i=\left\lceil\frac{k}{2}\right\rceil-1, \\
k-t+1 & \text { if } i=\left\lceil\frac{k}{2}\right\rceil,\left\lceil\frac{k}{2}\right\rceil+1, \ldots, k-1 .\end{cases}
\end{aligned}
$$

Let $X_{i}=\left(G_{1}-E(W)\right)\left[\left\{a_{i}\right\} \cup B_{1}\right]$ for $i=0,1, \ldots, k-1$. Then for even $t, X_{i}=S_{k-t}$, and for odd $t$,

$$
X_{i}= \begin{cases}S_{k-t-1} & \text { if } i=0,1, \ldots,\left\lceil\frac{k}{2}\right\rceil-2, \\ S_{k-t-\delta} & \text { if } i=\left\lceil\frac{k}{2}\right\rceil-1, \\ S_{k-t+1} & \text { if } i=\left\lceil\frac{k}{2}\right\rceil,\left\lceil\frac{k}{2}\right\rceil+1, \ldots, k-1\end{cases}
$$

with the center at $a_{i}$. In the following, we will show that $H$ can be decomposed into $r$ copies of $S_{k}$ with centers in $B_{2}$, and into $k$ copies of $S_{t}$ with centers in $A_{1}$ for even $t$, and into $\lceil k / 2\rceil-1$ copies of $S_{t+1}$ with centers in $\left\{a_{0}, a_{1}, \ldots, a_{\lceil k / 2\rceil-2}\right\}$, an $S_{t+\delta}$ with the center at $a_{\lceil k / 2\rceil-1}$, and $k-\lceil k / 2\rceil$ copies of $S_{t-1}$ with centers in $\left\{a_{\lceil k / 2\rceil}, a_{\lceil k / 2\rceil+1}, \ldots, a_{k-1}\right\}$ for odd $t$.

We show the required star decomposition of $H$ by orienting the edges of $H$. For any vertex $x$ of $H$, the outdegree $\operatorname{deg}_{H}^{+} x$ (indegree $\operatorname{deg}_{H}^{-} x$, resp.) of $x$ in an orientation of $H$ is the number of arcs incident from (to, resp.) $x$. It is sufficient to show that there exists an orientation of $H$ such that

$$
\operatorname{deg}_{H}^{+} b_{j}=k,
$$

where $j=k, k+1, \ldots, k+r-1$, and for even $t$

$$
\operatorname{deg}_{H}^{+} a_{i}=t
$$

where $i=0,1, \ldots, k-1$, and for odd $t$

$$
\operatorname{deg}_{H}^{+} a_{i}= \begin{cases}t+1 \quad \text { if } i=0,1, \ldots,\left\lceil\frac{k}{2}\right\rceil-2, \\ t+\delta \quad \text { if } i=\left\lceil\frac{k}{2}\right\rceil-1, \\ t-1 \quad \text { if } i=\left\lceil\frac{k}{2}\right\rceil,\left\lceil\frac{k}{2}\right\rceil+1, \ldots, k-1 .\end{cases}
$$

We first consider the edges oriented outward from $A_{1}$ according to the parity of $t$. Let $\beta=k+(t+1)(\lceil k / 2\rceil-$ 1) and $\gamma=\beta+t+\delta$. If $t$ is even, then the edges $a_{i} b_{k+t i}, a_{i} b_{k+t i+1}, \ldots, a_{i} b_{k+t i+t-1}$ are all oriented outward from $a_{i}$, where $i=0,1, \ldots, k-1$. If $t$ is odd, then the edges $a_{i} b_{k+(t+1) i}, a_{i} b_{k+(t+1) i+1}, \ldots, a_{i} b_{k+(t+1) i+t}$ for $i=0,1, \ldots,\lceil k / 2\rceil-$ 2 , and $a_{\lceil k / 2\rceil-1} b_{\beta}, a_{\lceil k / 2\rceil-1} b_{\beta+1}, \ldots, a_{\lceil k / 2\rceil-1} b_{\beta+t+\delta-1}$, as well as $a_{i} b_{(t-1)(i-\lceil k / 2\rceil)+\gamma}, a_{i} b_{(t-1)(i-\lceil k / 2\rceil)+\gamma+1}, \ldots, a_{i} b_{(t-1)(i-\lceil k / 2\rceil)+\gamma+t-2}$ for $i=\lceil k / 2\rceil,\lceil k / 2\rceil+1, \ldots, k-1$ are all oriented outward from $a_{i}$. In both cases, the subscripts of $b$ are taken modulo $r$ in the set of numbers $\{k, k+1, \ldots, k+r-1\}$. Note that for even $t$ we orient $t$ edges from each $a_{i}$ and for odd $t$ we orient at most $t+1$ edges from $a_{i}$. By inequality (4), we have $t+1 \leq r$, which assures us that there are enough edges for the above orientation.

Finally, the edges which are not oriented yet are all oriented from $B_{2}$ to $A$. From the construction of the orientation, it is easy to see that (9) and (10) are satisfied, and for all $b_{w}, b_{w^{\prime}} \in B_{2}$, we have

$$
\left|\operatorname{deg}_{H}^{-} b_{w}-\operatorname{deg}_{H}^{-} b_{w^{\prime}}\right| \leq 1
$$

So, we only need to check (8).

Since $\operatorname{deg}_{H}^{+} b_{w}+\operatorname{deg}_{H}^{-} b_{w}=k+r$ for $b_{w} \in B_{2}$, it follows from (11) that

$$
\left|\operatorname{deg}_{H}^{+} b_{w}-\operatorname{deg}_{H}^{+} b_{w^{\prime}}\right| \leq 1
$$

for $b_{w}, b_{w^{\prime}} \in B_{2}$. Note that for even $t, \sum_{i=0}^{k-1} \operatorname{deg}_{H}^{+} a_{i}=t k$, and for odd $t$,

$$
\begin{aligned}
& \sum_{i=0}^{k-1} \operatorname{deg}_{H}^{+} a_{i}=(t+1)\left(\left\lceil\frac{k}{2}\right\rceil-1\right)+t+\delta+(t-1)\left(k-\left\lceil\frac{k}{2}\right\rceil\right) \\
& =(t-1) k+2\left(\left\lceil\frac{k}{2}\right\rceil\right)-1+\delta \\
& = \begin{cases}(t-1) k+2\left(\frac{k}{2}\right)-1+1 & \text { if } k \text { is even, } \\
(t-1) k+\frac{2(k+1)}{2}-1 & \text { if } k \text { is odd. }\end{cases} \\
& =t k \text {. }
\end{aligned}
$$


Thus,

$$
\begin{aligned}
\sum_{w=k}^{k+r-1} \operatorname{deg}_{H}^{+} b_{w} & =\left|E\left(K_{k+r, r}\right)\right|-\sum_{i=0}^{k-1} \operatorname{deg}_{H}^{+} a_{i} \\
& =(k+r) r-t k \\
& =k r+r^{2}-r^{2} \\
& =k r .
\end{aligned}
$$

Therefore from (12), we have $\operatorname{deg}_{H}^{+} b_{w}=k$ for $b_{w} \in B_{2}$. This establishes (8). Hence, there exists the required decomposition $\mathscr{D}$ of $H$. Let $X_{i}^{\prime}$ be the star with center at $a_{i}$ in $\mathscr{D}$ for $i=0,1, \ldots, k-1$. Then, $X_{i}+X_{i}^{\prime}$ is a $k$-star. This completes the proof.

Now, we are ready for the main result. It is obtained form the arguments above, Lemma 4 and Lemmas 3, 4, 5, and 6.

Theorem 7. Let $k$ and $n$ be positive integers. Then, $K_{n, n}$ has $a\left(P_{k}, S_{k}\right)$-multidecomposition if and only if $k \leq n$ and $n^{2} \equiv$ $0(\bmod k)$.

\section{Acknowledgment}

The authors are grateful to the referees for the valuable comments.

\section{References}

[1] A. Bouchet and J.-L. Fouquet, "Trois types de décompositions d'un graphe en chaînes," Annals of Discrete Mathematics, vol. 17, pp. 131-141, 1983.

[2] K. Heinrich, J. Liu, and M. Yu, " $P_{4}$-decompositions of regular graphs," Journal of Graph Theory, vol. 31, no. 2, pp. 135-143, 1999.

[3] M. S. Jacobson, M. Truszczyński, and Z. Tuza, "Decompositions of regular bipartite graphs," Discrete Mathematics, vol. 89, no. 1, pp. 17-27, 1991.

[4] A. Kotzig, "From the theory of finite regular graphs of degree three and four," Časopis pro pěstování matematiky, vol. 82, pp. 76-92, 1957.

[5] C. S. Kumar, "On $P_{4}$-decomposition of graphs," Taiwanese Journal of Mathematics, vol. 7, no. 4, pp. 657-664, 2003.

[6] H.-C. Lee, M.-J. Lee, and C. Lin, "Isomorphic path decompositions of $\lambda K_{n, n, n}\left(\lambda K_{n, n, n}^{x}\right)$ for odd $n$," Taiwanese Journal of Mathematics, vol. 13, no. 2A, pp. 393-402, 2009.

[7] C. A. Parker, Complete bipartite graph path decompositions [Ph.D. thesis], Auburn University, Auburn, Ala, USA, 1998.

[8] T.-W. Shyu, "Path decompositions of $\lambda K_{n, n}$ " Ars Combinatoria, vol. 85, pp. 211-219, 2007.

[9] T.-W. Shyu and C. Lin, "Isomorphic path decompositions of crowns," Ars Combinatoria, vol. 67, pp. 97-103, 2003.

[10] M. Tarsi, "Decomposition of a complete multigraph into simple paths: nonbalanced handcuffed designs," Journal of Combinatorial Theory A, vol. 34, no. 1, pp. 60-70, 1983.

[11] M. Truszczyński, "Note on the decomposition of $\lambda K_{m, n}\left(\lambda K_{m, n}^{*}\right)$ into paths," Discrete Mathematics, vol. 55, no. 1, pp. 89-96, 1985.

[12] K. Heinrich, "Path-decompositions," Le Matematiche, vol. 47, no. 2, pp. 241-258, 1992.
[13] D. E. Bryant, S. El-Zanati, C. V. Eynden, and D. G. Hoffman, "Star decompositions of cubes," Graphs and Combinatorics, vol. 17, no. 1, pp. 55-59, 2001.

[14] C. Lin, J.-J. Lin, and T.-W. Shyu, "Isomorphic star decompositions of multicrowns and the power of cycles," Ars Combinatoria, vol. 53, pp. 249-256, 1999.

[15] M. Tarsi, "Decomposition of complete multigraphs into stars," Discrete Mathematics, vol. 26, no. 3, pp. 273-278, 1979.

[16] S. Tazawa, "Decomposition of a complete multipartite graph into isomorphic claws," Society for Industrial and Applied Mathematics, vol. 6, no. 3, pp. 413-417, 1985.

[17] K. Ushio, S. Tazawa, and S. Yamamoto, "On clawdecomposition of a complete multipartite graph," Hiroshima Mathematical Journal, vol. 8, no. 1, pp. 207-210, 1978.

[18] S. Yamamoto, H. Ikeda, S. Shige-eda, K. Ushio, and N. Hamada, "On claw-decomposition of complete graphs and complete bigraphs," Hiroshima Mathematical Journal, vol. 5, pp. 33-42, 1975.

[19] A. Abueida and M. Daven, "Multidesigns for graph-pairs of order 4 and 5," Graphs and Combinatorics, vol. 19, no. 4, pp. 433447, 2003.

[20] A. Abueida and M. Daven, "Multidecompositions of the complete graph," Ars Combinatoria, vol. 72, pp. 17-22, 2004.

[21] A. Abueida and M. Daven, "Multidecompositions of several graph products," Graphs and Combinatorics, 2012.

[22] A. Abueida and T. O'Neil, "Multidecomposition of $\lambda K_{m}$ into small cycles and claws," Bulletin of the Institute of Combinatorics and its Applications, vol. 49, pp. 32-40, 2007.

[23] H. M. Priyadharsini and A. Muthusamy, “ $\left(G_{m}, H_{m}\right)$-multifactorization of $\lambda K_{m}$," Journal of Combinatorial Mathematics and Combinatorial Computing, vol. 69, pp. 145-150, 2009.

[24] T.-W. Shyu, "Decomposition of complete graphs into paths and stars," Discrete Mathematics, vol. 310, no. 15-16, pp. 2164-2169, 2010.

[25] T.-W. Shyu, "Decompositions of complete graphs into paths and cycles," Ars Combinatoria, vol. 97, pp. 257-270, 2010.

[26] T. W. Shyu, "Decomposition of complete graphs into paths of length three and triangles," Ars Combinatoria, vol. 107, pp. 209224, 2012.

[27] T.-W. Shyu, "Decomposition of complete graphs into cycles and stars," Graphs and Combinatorics, 2011.

[28] H.-C. Lee, "Multidecompositions of complete bipartite graphs into cycles and stars," Ars Combinatoria, vol. 108, pp. 355-364, 2013. 


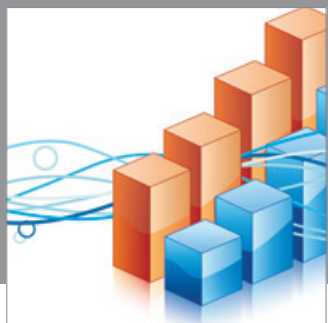

Advances in

Operations Research

mansans

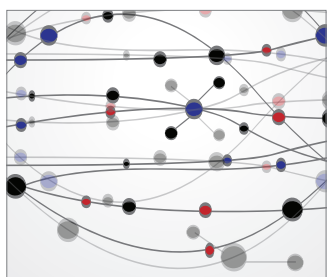

The Scientific World Journal
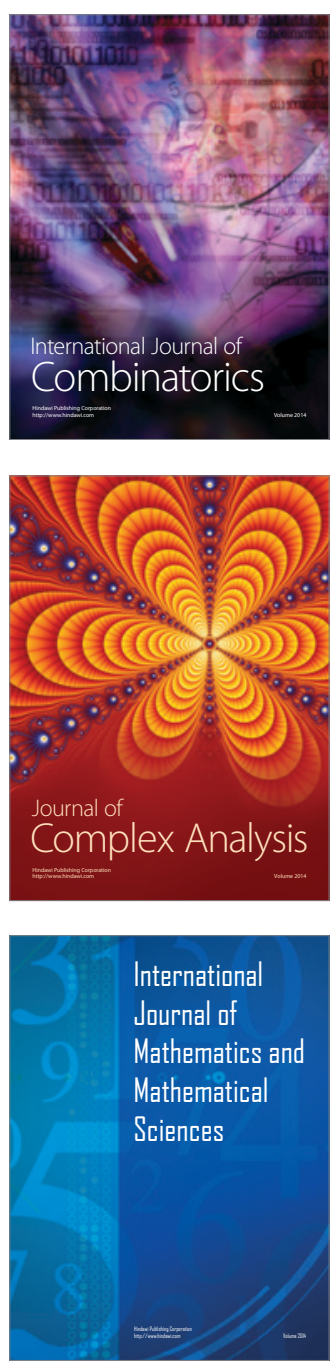
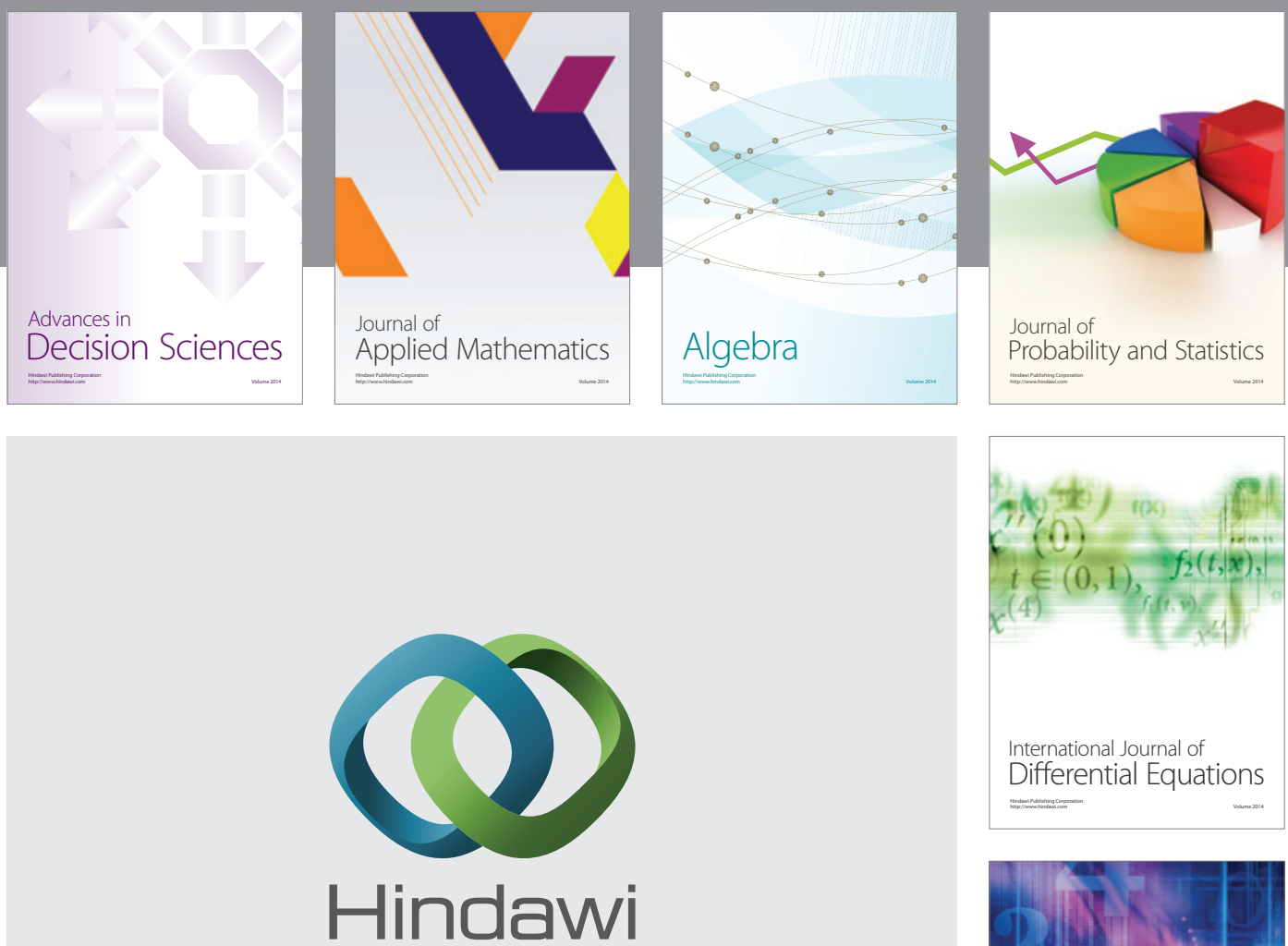

Submit your manuscripts at http://www.hindawi.com
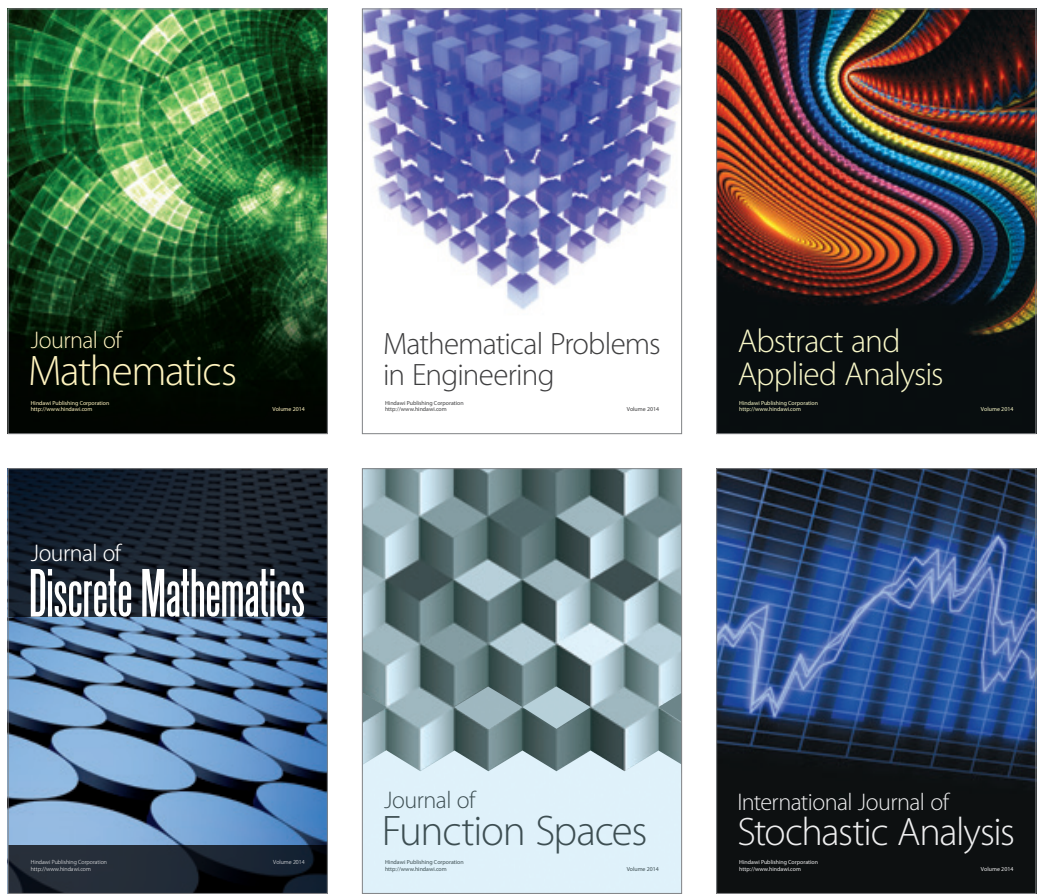

Journal of

Function Spaces

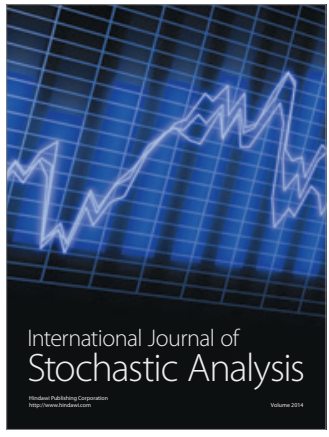

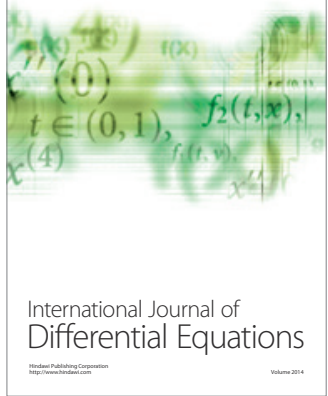
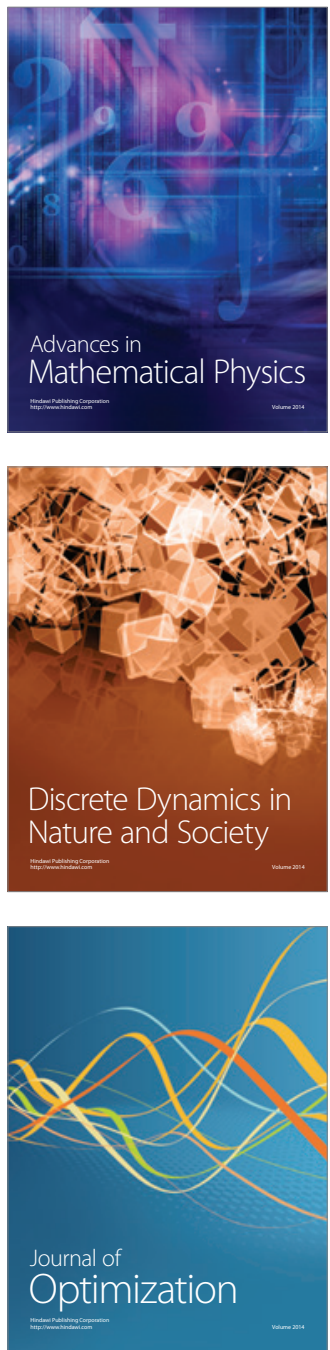